\title{
Belief Reasoning, Revision and Fusion by Matrix Algebra
}

\author{
Churn-Jung Liau \\ Institute of Information Science \\ Academia Sinica, Taipei, Taiwan \\ liaucj@iis.sinica.edu.tw
}

\begin{abstract}
Representation of belief states is an important issue for knowledge based systems. In this paper, we develop a matrix representation for ordered belief states and show that belief reasoning, revision and fusion can all be interpreted as operations of matrix algebra. Thus, the matrix representation can serve as the basis of algebraic semantics for belief logic.
\end{abstract}

Key Words: Belief states, matrix algebra, belief reasoning, belief revision, belief fusion, multi-agent systems.

\section{Introduction}

Knowledge representation is crucially important to knowledge-based applications. While we expect proper knowledge to be true, the term "knowledge" used in knowledge-based systems usually refers to information and belief in a broad sense. Therefore, knowledge can be fallible information in such applications. Consequently, the representation of belief states plays a key role in knowledge-based systems.

In many domains, such as epistemic reasoning $[10,11]$, belief revision[1, 14, 15] and belief fusion[2, 6, 16, 18-21,23,27], a knowledge base or a belief base consists of a set of sentences in a logical language. Since a belief base can be identified with the set of models satisfying it, an agent's belief state is usually represented as a subset of possible worlds.

However, some more finely tuned representations have been proposed, such as total pre-orders over the set of possible worlds $[4,7,17,24]$, ordinal conditional functions $[5,26,28]$, possibility distributions $[3,8,9]$, belief functions [25], and pedigreed belief states[12,13]. One of the most popular representation formalisms is an ordering over the set of possible worlds.

A natural representation of an ordering can be achieved by using a Boolean matrix, if the set of possible worlds is finite. In this paper, we adopt such a representation and show that belief reasoning, revision and fusion can be interpreted in matrix algebra. Thus, the matrix representation can serve as the basis of algebraic semantics for belief logic. 


\section{Matrix Representation of Belief States}

In this paper, $A, B$ and $M$ (possibly with subscripts) denote real-valued matrices. If $M$ is a $n \times m$ matrix, then for $0 \leq i \leq n-1$ and $0 \leq j \leq m-1, M(i, j), M(i, \cdot)$ and $M(\cdot, j)$ denote the element in the $(i, j)$ entry, the $i$-th row vector and the $j$-th column vector of the matrix respectively. Usually, we also write $M(i)$ and $M(j)$ for the row vector and the column vector if they are clear from the context. A Boolean matrix is a real-valued matrix whose elements all belong to the set $\{0,1\}$. Also, let $I, \mathbf{1}$ and $\mathbf{0}$ denote the unit square matrix, complete matrix and zero matrix respectively. In other words, $I, \mathbf{1}$ and $\mathbf{0}$ are Boolean matrices such that $I(i, j)=1$ iff $i=j, \mathbf{1}(i, j)=1$ and $\mathbf{0}(i, j)=0$ for all $i$ and $j$. The dimensions (i.e., numbers of rows and columns) of $I, \mathbf{1}$ and $\mathbf{0}$ depend on the context. If necessary, we will write $I_{n \times m}, \mathbf{1}_{n \times m}$, or $\mathbf{0}_{n \times m}$ to indicate their dimensions. Furthermore, we will identify a $1 \times 1$ matrix with its element.

The following operations of matrix algebra are extensively used in this paper.

Definition 1 Let $A$ and $B$ be two matrices and $r$ is a real number. The following binary operations can be defined when $A$ and $B$ have the same dimensions:

1. $\operatorname{Max}:(A \vee B)(i, j)=\max (A(i, j), B(i, j))$,

2. Min: $(A \wedge B)(i, j)=\min (A(i, j), B(i, j))$,

3. Sum: $(A+B)(i, j)=A(i, j)+B(i, j)$,

4. Difference: $(A-B)(i, j)=A(i, j)-B(i, j)$,

5. Scalar product: $(r \cdot A)(i, j)=r \cdot A(i, j)$.

When the column number of $A$ is equal to the row number of $B$, another binary operation, the (Boolean) product, can be defined as:

$$
(A \cdot B)(i, j)=\max _{0 \leq k \leq n-1} \min (A(i, k), B(k, j))
$$

where $n$ is the column number of $A$. Let $m \geq 0$, then $A^{m}$ is inductively defined as $A^{m-1} \cdot A$ where $A^{0}=I$. Furthermore, there are also several unary operations:

1. Transposition: $A^{t}(i, j)=A(j, i)$,

2. Transitive closure: $A^{+}=\bigvee_{m \geq 1} A^{m}$

3. Indicator: $A^{d}(i, j)=\left\{\begin{array}{l}1, \text { if } A(i, j)>0 \text { and } A(i, j) \geq A(j, i) \text {, } \\ 0, \text { otherwise. }\end{array}\right.$

If $\Phi_{0}=\{p, q, \cdots\}$ is a finite set of atomic propositions and $\mathcal{L}$ is the propositional language based on $\Phi_{0}$, then a possible world is a truth assignment $w: \Phi_{0} \rightarrow\{0,1\}$. As usual, the domain of $w$ can be extended to the set of all well-formed formulas (wffs) in $\mathcal{L}$. If $\mathcal{W}=\left\{w_{0}, w_{1}, \cdots, w_{n-1}\right\}$ denotes the set of all possible worlds, then a belief state is defined as an $n \times n$ Boolean matrix $B$. Intuitively, a belief state $B$ defines an associated ordering relation $\prec_{B}$ over 
$\mathcal{W}$ such that $B(i, j)=1$ iff $w_{j} \prec_{B} w_{i}$. For each wff $\varphi$, the characteristic matrix of $\varphi$ is the $n \times n$ Boolean matrix $M_{\varphi}$ defined by

$$
M_{\varphi}(i, j)=\left\{\begin{array}{l}
1, \text { if } i=j \text { and } w_{i}(\varphi)=1 \\
0, \text { otherwise. }
\end{array}\right.
$$

Note that by this definition, we have the equations:

$$
\begin{aligned}
M_{\varphi \vee \psi} & =M_{\varphi} \vee M_{\psi} \\
M_{\varphi \wedge \psi} & =M_{\varphi} \wedge M_{\psi} \\
M_{\neg \varphi} & =I-M_{\varphi}
\end{aligned}
$$

where $I$ is the $n \times n$ unit matrix.

\section{Belief Reasoning, Revision and Fusion}

Given the matrix representation of belief states and wffs, we can implement the tasks of belief reasoning, revision and fusion using matrix algebra.

\subsection{Belief reasoning}

Definition 2 Given a belief state $B$ and two wffs $\varphi$ and $\psi$, we say that $\varphi$ preferentially implies $\psi$ under $B$, denoted by $\varphi \models_{B} \psi$, iff

$$
\left(\mathbf{1}_{1 \times n}-\mathbf{1}_{1 \times n} \cdot M_{\psi}\right) \cdot\left[\mathbf{1}_{n \times 1}-\left(M_{\varphi} \cdot B \cdot M_{\varphi} \vee M_{\neg \varphi}\right) \cdot \mathbf{1}_{n \times 1}\right]=0,
$$

where we assume has precedence over other binary operations.

The intuition of the above definition can be seen by interpreting a belief state as an ordering over possible worlds. Let $\prec$ be a binary relation over $\mathcal{W}$ and $X \subseteq \mathcal{W}$, then the choice set [12] of $X$ with respect to $\prec$ is

$$
C(X, \prec)=\left\{w \in X: \nexists w^{\prime} \in X, w^{\prime} \prec w\right\} .
$$

Let the truth set of a wff $\varphi$ be defined as $|\varphi|=\{w \in \mathcal{W}: w(\varphi)=1\}$, then the set of minimal models of $\varphi$ with respect to $\prec$, denoted by $\operatorname{Min}(\varphi, \prec)$, is equal to $C(|\varphi|, \prec)$. The following proposition states the relationship between the preferential implication and minimal model belief reasoning.

Proposition 1 Let $B$ be a belief state and $\prec_{B}$ be its associated ordering, then for any wffs $\varphi$ and $\psi$

$$
\varphi \mid=_{B} \psi \text { iff } \operatorname{Min}\left(\varphi, \prec_{B}\right) \subseteq|\psi| .
$$

Proof: Let

$$
\begin{gathered}
A_{1}=M_{\varphi} \cdot B \cdot M_{\varphi} \vee M_{\neg \varphi}, \\
A_{2}=\mathbf{1}_{n \times 1}-A_{1} \cdot \mathbf{1}_{n \times 1}, \\
A_{3}=\mathbf{1}_{1 \times n}-\mathbf{1}_{1 \times n} \cdot M_{\psi}
\end{gathered}
$$

then $A_{1}(i, j)=1$ iff one of the following two conditions holds: 
(i) $w_{i} \models \varphi, w_{j} \models \varphi$ and $w_{j} \prec_{B} w_{i}$, or

(ii) $i=j$ and $w_{i} \not \models \varphi$.

Thus, $\max _{0 \leq j \leq n-1} A(i, j)=1$ iff $w_{i} \notin \operatorname{Min}\left(\varphi, \prec_{B}\right)$. This results in $A_{2}(i, 0)=1$ iff $w_{i} \in \operatorname{Min}\left(\varphi, \prec_{B}\right)$. On the other hand , $A_{3}(0, i)=1$ iff $w_{i} \not \models \psi$. Therefore,

$$
\begin{aligned}
& \operatorname{Min}\left(\varphi, \prec_{B}\right) \subseteq|\psi| \\
\Leftrightarrow & \nexists 0 \leq i \leq n-1, w_{i} \in \operatorname{Min}\left(\varphi, \prec_{B}\right) \text { and } w_{i} \not \models \psi \\
\Leftrightarrow & A_{3} \cdot A_{2}=\max _{0 \leq i \leq n-1} \min \left(A_{3}(1, i), A_{2}(i, 1)\right)=0 \\
\Leftrightarrow & \varphi \models_{B} \psi .
\end{aligned}
$$

Note that we do not impose any restriction on the matrix representation of belief states, so minimal models of a wff $\varphi$ may not exist. Some reasonable restrictions have been proposed, such as transitivity and modularity[12]. However, our definition can still be used in the more restricted form of belief states.

\subsection{Belief revision}

Definition 3 Let $B$ be a belief state and $\varphi$ be a wff, then the revised state of $B$ by $\varphi$ is defined as

$$
B^{\circ} \varphi=\left[B \vee\left(M_{\neg \varphi} \cdot \mathbf{1} \wedge \mathbf{1} \cdot M_{\varphi}\right)\right] \wedge\left(M_{\neg \varphi} \cdot \mathbf{1} \vee \mathbf{1} \cdot M_{\varphi}\right),
$$

where the dimension of $\mathbf{1}$ is $n \times n$.

This definition corresponds to a natural approach to revise an ordering over possible worlds with a wff. Let $\prec$ be an ordering relation over $\mathcal{W}$, then revising $\prec$ with a wff $\varphi$ results in a new ordering $\prec^{\prime}=\operatorname{Rev}(\prec, \varphi)$ defined by

$$
\begin{aligned}
w \prec^{\prime} w^{\prime} \Leftrightarrow w(\varphi) & =1 \text { and } w^{\prime}(\varphi)=0, \text { or } \\
w(\varphi) & =w^{\prime}(\varphi) \text { and } w \prec w^{\prime} .
\end{aligned}
$$

According to this definition, after the revision, all possible worlds satisfying $\varphi$ are preferred over those that do not, whereas the ordering between other worlds is kept intact. The following proposition shows the equivalence between these two definitions.

Proposition 2 Let $B$ be a belief state and $\varphi$ be a wff, then

$$
\operatorname{Rev}\left(\prec_{B}, \varphi\right)=\prec_{B^{\circ} \varphi}
$$

where $\prec_{B}$ and $\prec_{B^{\circ} \varphi}$ are, respectively, the associated ordering of $B$ and $B^{\circ} \varphi$.

Proof: First, we note that for all $0 \leq i \leq n-1,\left(M_{\neg \varphi} \cdot \mathbf{1}\right)(i, j)=1$ iff $w_{j}(\varphi)=0$ and for all $0 \leq j \leq n-1,\left(\mathbf{1} \cdot M_{\neg \varphi}\right)(i, j)=1$ iff $w_{i}(\varphi)=1$. Let $A_{1}=B \vee\left(M_{\neg \varphi}\right.$. $\left.\mathbf{1} \wedge \mathbf{1} \cdot M_{\varphi}\right)$ and $A_{2}=M_{\neg \varphi} \cdot \mathbf{1} \vee \mathbf{1} \cdot M_{\varphi}$, then

$$
A_{1}(i, j)=1 \Leftrightarrow B(i, j)=1 \vee\left(w_{i}(\varphi)=0 \wedge w_{j}(\varphi)=1\right)
$$


and

$$
\begin{aligned}
A_{2}(i, j)=1 & \Leftrightarrow w_{i}(\varphi)=0 \vee w_{j}(\varphi)=1 \\
& \Leftrightarrow w_{i}(\varphi)=w_{j}(\varphi) \vee\left(w_{i}(\varphi)=0 \wedge w_{j}(\varphi)=1\right) .
\end{aligned}
$$

Therefore,

$$
\begin{aligned}
B^{\circ} \varphi(i, j)=1 & \Leftrightarrow A_{1}(i, j)=1 \wedge A_{2}(i, j)=1 \\
& \Leftrightarrow\left(w_{i}(\varphi)=0 \wedge w_{j}(\varphi)=1\right) \vee\left(w_{i}(\varphi)=w_{j}(\varphi) \wedge B(i, j)=1\right) .
\end{aligned}
$$

This is equivalent to

$$
w_{j} \prec_{B^{\circ} \varphi} w_{i} \Leftrightarrow\left(w_{i}(\varphi)=0 \wedge w_{j}(\varphi)=1\right) \vee\left(w_{i}(\varphi)=w_{j}(\varphi) \wedge w_{j} \prec_{B} w_{i}\right),
$$

so we have $\prec_{B^{\circ} \varphi}=\operatorname{Rev}\left(\prec_{B}, \varphi\right)$.

An alternative revision strategy, called natural revision, has been proposed in [4]. In that operator, only minimal possible worlds satisfying $\varphi$ in the original belief state are preferred over others. Consequently, more possible worlds remain intact in natural revision. Formally, a natural revision of $\prec$ with a wff $\varphi$ results in a new ordering $\prec^{\prime}=N \operatorname{Rev}(\prec, \varphi)$ defined by ${ }^{1}$

1. if $w \in \operatorname{Min}(\varphi, \prec)$, then $w \prec^{\prime} v$ for any $v \notin \operatorname{Min}(\varphi, \prec)$ and

2. if $v, w \notin \operatorname{Min}(\varphi, \prec)$, then $w \prec^{\prime} v$ iff $w \prec v$.

The natural revision operator can be modelled in the matrix representation by the following definition.

Definition 4 Let $B$ be a belief state and $\varphi$ be a wff. Define the column vector (i.e. $n \times 1$ matrices) a and the row vector(i.e. $1 \times n$ matrices) $\mathbf{b}$ as follows:

$$
\begin{gathered}
\mathbf{a}=\left(M_{\varphi} \cdot B \cdot M_{\varphi} \vee M_{\neg \varphi}\right) \cdot \mathbf{1}_{n \times 1} \\
\mathbf{b}=\left(\mathbf{1}_{n \times 1}-\mathbf{a}\right)^{t} .
\end{gathered}
$$

Then, the naturally revised state of $B$ by $\varphi$ is defined as

$$
B^{*} \varphi=\left[B \vee\left(\mathbf{1}_{n \times 1} \cdot \mathbf{b}\right)\right] \wedge\left(\mathbf{a} \cdot \mathbf{1}_{1 \times n}\right) .
$$

Proposition 3 Let $B$ be a belief state and $\varphi$ be a wff, then

$$
N \operatorname{Rev}\left(\prec_{B}, \varphi\right)=\prec_{B^{*} \varphi}
$$

where $\prec_{B}$ and $\prec_{B * a \varphi}$ are respectively the associated ordering of $B$ and $B^{*} \varphi$.

\footnotetext{
${ }^{1}$ In [4], natural revision operator is only applied to belief states represented by total pre-orders, so the definition here is a slight generalization of that given in [4].
} 
Proof: Let $A=M_{\varphi} \cdot B \cdot M_{\varphi} \vee M_{\neg \varphi}$, then

$$
\begin{gathered}
A(i, j)=1 \Leftrightarrow w_{i}(\varphi)=w_{j}(\varphi)=1 \text { and } w_{j} \prec_{B} w_{i}, \text { or } \\
i=j \text { and } w_{i}(\varphi)=0 .
\end{gathered}
$$

Thus, $\mathbf{a}(i, 0)=\max _{0 \leq j \leq n-1} A(i, j)=1$ iff $w_{i} \notin \operatorname{Min}\left(\varphi, \prec_{B}\right)$ and $\mathbf{b}(0, j)=1$ iff $w_{j} \in \operatorname{Min}\left(\varphi, \prec_{B}\right)$. Therefore, $B^{*} \varphi(i, j)=1$ iff the following two conditions are simultaneously satisfied:

1. $w_{j} \prec_{B} w_{i}$ or $w_{j} \in \operatorname{Min}\left(\varphi, \prec_{B}\right)$;

2. $w_{i} \notin \operatorname{Min}\left(\varphi, \prec_{B}\right)$.

Consequently, we have

$$
\begin{aligned}
w_{j} \prec_{B^{*} \varphi} w_{i} \Leftrightarrow & w_{j} \in \operatorname{Min}\left(\varphi, \prec_{B}\right) \text { and } w_{i} \notin \operatorname{Min}\left(\varphi, \prec_{B}\right) \text { or } \\
& w_{j} \prec_{B} w_{i} \text { and } w_{i}, w_{j} \notin \operatorname{Min}\left(\varphi, \prec_{B}\right),
\end{aligned}
$$

so $N \operatorname{Rev}\left(\prec_{B}, \varphi\right)=\prec_{B^{*} \varphi}$ by the definition.

\subsection{Belief fusion}

In belief fusion, the notion of pedigreed belief states proposed in $[12,13]$ is a very general one. The advantage of the pedigreed belief state representation is that it can keep track of information about the credibility of sources. By the matrix notation, we can easily represent a pedigreed belief state and the belief state induced from it. Let $\Omega$ be a set of information sources, then it is assumed that for each source $s \in \Omega$, there is a belief state $B_{s}$ associated with it. It is also assumed that the credibility of information sources is determined by a ranking function rank $: \Omega \rightarrow \mathcal{N}$, where $\mathcal{N}$ is the set of positive integers.

Definition 5 Let $\Omega$ be a set of information sources and rank: $\Omega \rightarrow \mathcal{N}$ be its ranking function, then

1. the pedigreed belief state of qualitatively fusing $\Omega$ is

$$
P B_{q}(\Omega)=\bigvee_{s \in \Omega} \operatorname{rank}(s) \cdot B_{s}
$$

2. the pedigreed belief state of arithmetically fusing $\Omega$ is

$$
P B_{a}(\Omega)=\sum_{s \in \Omega} \operatorname{rank}(s) \cdot B_{s}
$$

3. the pedigreed belief state of exponentially fusing $\Omega$ is

$$
P B_{e}(\Omega)=\sum_{s \in \Omega} \alpha^{r a n k(s)-1} \cdot B_{s},
$$

where $\alpha \geq|\Omega|$ is a real number, 
4. the belief state of fusing $\Omega$ (qualitatively, arithmetically, or exponentially) is

$$
\oplus_{x}(\Omega)=\left(P B_{x}(\Omega)\right)^{d}
$$

where $x=q$, a or $e$.

The definition of qualitative fusion is taken from [12]. In that work, a generalized belief state is defined as a modular and transitive relation over $\mathcal{W}$. A relation $\prec$ over $\mathcal{W}$ is transitive if $w_{1} \prec w_{2}$ and $w_{2} \prec w_{3}$ imply $w_{1} \prec w_{3}$ and modular if $w_{1} \prec w_{2}$ implies that for any $w \in \mathcal{W}, w \prec w_{2}$ or $w_{1} \prec w$. Therefore, each information source $s$ is associated with a generalized belief state $\prec^{s}$ and an aggregation operator $A G R R f$ is defined for a set of information sources $S$ as

$$
\operatorname{AGRRf}(S)=\left\{(w, v) \mid \exists s \in S . w \prec^{s} v \wedge\left(\forall s^{\prime} \sqsupset s \in S . w \approx^{s^{\prime}} v\right)\right\}
$$

where $s^{\prime} \sqsupset s$ means that $\operatorname{rank}\left(s^{\prime}\right)>\operatorname{rank}(s)$ and $w \approx^{s^{\prime}} v$ means that $\neg\left(w \prec s^{\prime}\right.$ $v) \wedge \neg\left(v \prec s^{\prime} w\right)$. The pedigreed belief state for a set of information sources $S$ is then defined as a pair $(\prec, l)$, where $\prec=A G R R f(S)$ and $l: \prec \rightarrow \mathcal{N}$ such that

$$
l((w, v))=\max \left\{\operatorname{rank}(s) \mid w \prec^{s} v, s \in S\right\} .
$$

Note that in our definition, each information source is associated with a belief state in matrix form, whereas in [12], it is associated with a generalized belief state in the form of a modular and transitive relation. Let $\Omega$ denote a set of information sources in our definition such that each $\prec_{B_{s}}$ is transitive and modular. Then, we can define $S_{\Omega}$ as the corresponding set of information sources in the sense of [12], so that each $s \in S_{\Omega}$ is associated with the ordering $\prec_{B_{s}}$.

Proposition 4 Let $\Omega$ be a set of information sources in our definition, then

$$
\operatorname{AGRRf}\left(S_{\Omega}\right)=\prec_{\oplus_{q}(\Omega)}
$$

and for each $0 \leq i, j \leq n-1$ such that $\oplus_{q}(\Omega)(i, j)=1$,

$$
l\left(\left(w_{j}, w_{i}\right)\right)=P B_{q}(\Omega)(i, j) .
$$

\section{Proof:}

1. On the one hand, using the definition of indicator operation, for all $0 \leq i, j \leq$ $n-1, w_{j} \prec_{\oplus_{q}(\Omega)} w_{j}$ iff $P B_{q}(\Omega)(i, j)>0$ and $P B_{q}(\Omega)(i, j) \geq P B_{q}(\Omega)(j, i)$. Since $P B_{q}(\Omega)(i, j)=\max _{s \in \Omega} \operatorname{rank}(s) \cdot B_{s}(i, j), P B_{q}(\Omega)(i, j)>0$ implies $\exists s \in S, B_{s}(i, j)=1$, i.e. $\exists s \in S, w_{j} \prec_{B_{s}} w_{i}$. Let $s_{0}$ denote such an information source such that $\operatorname{rank}\left(s_{0}\right)=P B_{q}(\Omega)(i, j)$, then $P B_{q}(\Omega)(i, j) \geq$ $P B_{q}(\Omega)(j, i)$ implies $\forall s \sqsupset s_{0}, B_{s}(i, j)=B_{s}(j, i)=0$, i.e. $\forall s \sqsupset s_{0}, w_{j} \approx^{s} w_{i}$. Therefore, $w_{j} \prec_{\oplus_{q}(\Omega)} w_{j}$ implies $\left(w_{j}, w_{i}\right) \in \operatorname{AGRRf}\left(S_{\Omega}\right)$. On the other hand , if $\left(w_{j}, w_{i}\right) \in \operatorname{AGRRf}\left(S_{\Omega}\right)$, then there exists $s \in \Omega$ such that $B_{s}(i, j)=1$ and for all $s^{\prime}$ such that $\operatorname{rank}\left(s^{\prime}\right)>\operatorname{rank}(s), B_{s^{\prime}}(j, i)=B_{s^{\prime}}(i, j)=0$. Therefore, $P B_{q}(\Omega)(i, j)>0$ and $P B_{q}(\Omega)(i, j) \geq P B_{q}(\Omega)(j, i)$ hold. This in turn implies $w_{j} \prec_{\oplus_{q}(\Omega)} w_{j}$. 


$$
\begin{aligned}
& \text { 2. } P B_{q}(\Omega)(i, j)=\max _{s \in \Omega} \operatorname{rank}(s) \cdot B_{s}(i, j)=\max \left\{\operatorname{rank}(s) \mid w_{j} \prec_{B_{s}} w_{i}, s \in\right. \\
& \Omega\}=l\left(\left(w_{j}, w_{i}\right)\right)
\end{aligned}
$$

While the operator $A G R R f(S)$ preserves the modularity of the generalized belief states associated with sources in $S$, it does not preserve the transitivity. The resultant belief state for the fusion is, in fact, the transitive closure of $A G R R f(S)$. Therefore, the definition of qualitative fusion is for a very general notion of belief states according to the matrix representation. If some additional properties (such as modularity and transitivity) are imposed on the definition of belief states, some further operations (such as transitive closure) are required for implementation of the belief fusion operator.

In [12], it is suggested that aggregation of sources based on a general framework of voting is also possible. While qualitative fusion accounts for the ranking of sources supporting or disagreeing with an opinion, it does not count the percentage of sources in each camp. The definition of arithmetic fusion implements the idea in a straightforward way by algebraic operations. When all sources are equally credible, this corresponds to merging by majority in the spirit of [22].

However, if the credibility of the sources are highly variant, it is possible that many sources of low credibility will outweigh one of high credibility. To circumvent this problem, we can use the exponential fusion. Since the base of the exponential terms is not less than the number of sources, it is guaranteed that the opinion of higher ranks will dominate all opinions of lower ranks.

\section{Conclusion}

In this paper, we show that matrix algebra can be applied to belief reasoning, revision and fusion in knowledge-based systems. By employing a Boolean matrix representation of belief states, the main tasks for belief or knowledge management in such systems can be reduced to algebraic operations in matrix algebra. This enables a uniform treatment of these reasoning tasks.

To make our approach general, we do not impose any restrictions on the matrix representation of belief states. However, in real applications, belief states are usually assumed to have some special structures, such as modularity and transitivity. In such cases, the closure of an algebraic operation with respect to these additional properties will become very important. In the further work, we will investigate how the closure property may influence the representation of belief states and the choice of algebraic operations.

Furthermore, the dimensions of matrices used in the representation of belief states are, in general, exponential with respect to the number of propositional variables. Since the dimensions of these matrices may be very large, it is impractical to implement reasoning tasks of belief logic directly via the semantic representation. Instead, a proof theory for belief logic should be developed and the matrix representation proposed in this paper can serve as the basis of an algebraic semantics for such logic. 


\section{Acknowledgements}

I would like to thank three anonymous referees for their valuable remarks.

\section{References}

1. C.E. Alchourrón, Gärdenfors, and D. Makinson. "On the logic of theory change: Partial meet contraction and revision functions". Journal of Symbolic Logic, 50:510-530, 1985.

2. C. Baral, S. Kraus, J. Minker, and V. S. Subrahmanian. "Combining knowledge bases consisting of first-order theories". Computational Intelligence, 8(1):45-71, 1992.

3. S. Benferhat, D. Dubois, and H. Prade. "From semantic to syntactic approaches to information combination in possibilistic logic". In B. Bouchon-Meunier, editor, Aggregation and Fusion of Imperfect Information, pages 141-161. Physica-Verlag, 1997.

4. C. Boutilier. "Revision sequences and nested conditionals". In Proceedings of the 13th International Joint Conference on Artificial Intelligence, pages 519-525, 1993.

5. C. Boutilier. "Generalized update: Belief change in dynamic settings". In Proceedings of the 14th International Joint Conference on Artificial Intelligence, pages 1550-1556, 1995.

6. L. Cholvy. "A logiccal approach to multi-souces reasoning". In M. Masuch and L. Pólos, editors, Knowledge Representation and Reasoning under Uncertainty, LNCS 808, pages 183-196. Springer-Verlag, 1994.

7. A. Darwiche and J. Pearl. "On the logic of iterated belief revision". Artificial Intelligence, 89(1):1-29, 1997.

8. D. Dubois and H. Prade. "Belief change and possibility theory". In P. Gärdenfors, editor, Belief Revision, pages 142-182. Cambridge University Press, 1992.

9. D. Dubois and H. Prade. "Possibility theory in information fusion". In Proc. of the Third International Conference on Information Fusion, pages TuA-1, 2000.

10. R. Fagin, J.Y. Halpern, Y. Moses, and M.Y. Vardi. Reasoning about Knowledge. MIT Press, 1996.

11. J.Y. Halpern and Y. Moses. "A guide to completeness and complexity for modal logics of knowledge and belief". Artificial Intelligence, 54:311-379, 1992.

12. P. Maynard-Reid II and D. Lehmann. "Representing and aggregating conflicting beliefs". In Proceedings of the 7th International Conference on Principles of Knowledge Representation and Reasoning, pages 153-164, 2000.

13. P. Maynard-Reid II and Y. Shoham. "Belief fusion: Aggregating pedigreed belief states". Journal of Logic, Language and Information, 10(2):183-209, 2001.

14. H. Katsuno and A. Medelzon. "On the difference between updating a knowledge base and revising it". In Proceedings of the Second International Conference on Principles of Knowledge Representation and Reasoning (KR'91), pages 387-394. Morgan Kaufmann Publisher, 1991.

15. H. Katsuno and A. Medelzon. "Propositional knowledge base revision and minimal change". Artificial Intelligence, 52:263-294, 1991.

16. S. Konieczny. "On the difference between merging knowledge bases and combining them". In Proceedings of the Seventh International Conference on Principles of Knowledge Representation and Reasoning (KR'00)., pages 135-144. Morgan Kaufmann Publisher, 2000. 
17. D. Lehmann. "Belief revision, revised". In Proceedings of the 14 th International Joint Conference on Artificial Intelligence, pages 1534-1540, 1995.

18. C.J. Liau. "A conservative approach to distributed belief fusion". In Proc. of the Third International Conference on Information Fusion, pages MoD4-1, 2000.

19. C.J. Liau. "Epistemic logics for information fusion". In T.D. Nielsen and N.L. Zhang, editors, Proc of the 7th European Conference on Symbolic and Quantitative Approaches to Reasoning with Uncertainty, LNAI 2711, pages 489-501. SpringerVerlag, 2003.

20. C.J. Liau. "A modal logic framework for multi-agent belief fusion". ACM Transactions on Computational Logic, 2004.

21. J. Lin. "Information sharing and knowledge merging in cooperative information systems". In Proceedings of the Fourth Workshop on Information Technologies and Systems, pages 58-66, 1994.

22. J. Lin and A.O. Mendelzon. "Knowledge base merging by majority". In R. Pareschi and B. Fronhoefer, editors, Dynamic Worlds: From the Frame Problem to Knowledge Management. Kluwer Academic Publisher, 1999.

23. S. Pradhan, J. Minker, and V. Subrahmanian. "Combining databases with prioritized information". Journal of Intelligent Information Systems, 4(3):231-260, 1995.

24. K. Segerberg. "Belief revision from the point of view of doxastic logic". Bull. of the IGPL, 3(4):535-553, 1995.

25. P. Smets. "Data fusion in the transferable belief model". In Proc. of the Third International Conference on Information Fusion, pages WeA-1, 2000.

26. W. Spohn. "Ordinal conditional functions: a dynamic theory of epistemic states". In W.L. Harper and B. Skyrms, editors, Causation in Decision, Belief Change, and Statistics, II, pages 105-134. Kluwer Academic Publishers, 1988.

27. V.S. Subrahmanian. "Amalgamating knowledge bases". ACM Transactions on Database Systems, 19(2):291-331, 1994.

28. M.A. Williams. "Transmutations of knowledge systems". In J. Doyle, E. Sandewall, and P. Torasso, editors, Proceedings of the 4 th International Conference on Principle of Knowledge Representation and Reasoning, pages 619-629. Morgan Kaufmann Publishers, 1994. 